\title{
An Investigation of the Grid Sensitivity in Large-Eddy Simulations of the Stable Boundary Layer
}

\author{
Björn Maronga ${ }^{1,2}$ D Dan $\mathrm{Li}^{3}$
}

Received: 15 January 2021 / Accepted: 2 August 2021 / Published online: 26 August 2021

(c) The Author(s) 2021

\begin{abstract}
We revisit the longstanding problem of grid sensitivity, i.e., the lack of grid convergence in large-eddy simulations (LES) of the stable boundary layer. We use a comprehensive set of LES of the well-known Global Energy and Water Cycle Experiment Atmospheric Boundary Layer Study 1 (GABLS1) case with varying grid spacings between $12.5 \mathrm{~m}$ and $1 \mathrm{~m}$ to investigate several physical processes and numerical features that are possible causes of grid sensitivity. Our results demonstrate that there are two resolution regimes in which grid sensitivity manifests differently. We find that changing the numerical advection schemes and the subgrid-scale models alters the simulation results, but the options tested do not fully address the grid-sensitivity issue. Moreover, sensitivity runs suggest that the surface boundary condition and the interaction of the surface with the near-surface flow, as well as the mixing with the free atmosphere, are unlikely to be the causes of the observed grid sensitivity. One interesting finding is that the grid sensitivity in the fine grid-spacing regime (grid spacings $\leq 2 \mathrm{~m}$ ) is closely related to the reduction in the energy content of large-scale turbulence, leading to less turbulence kinetic energy and hence lower boundary-layer heights. The present work demonstrates that there is still an urgent need to address this grid-sensitivity issue in order to perform reliable LES of the stable boundary layer.
\end{abstract}

Keywords GABLS1 · Grid sensitivity · Large-eddy simulation · PALM · Stable boundary layer

\section{Introduction}

The last two to three decades have witnessed a surge of studies using large-eddy simulations (LES) to investigate the atmospheric boundary layer and other high-Reynolds-number turbulent flows. In LES, the large-scale turbulence with spatial scales larger than the grid spacing $(\Delta)$ is resolved, while the effect of small-scale turbulence is parametrized using a subgrid-

Björn Maronga

bjorn.maronga@uib.no; maronga@muk.uni-hannover.de

1 Geophysical Institute, University of Bergen, Postbox 7803, 5020 Bergen, Norway

2 Institute of Meteorology and Climatology, Leibniz University Hannover, Hannover, Germany

3 Department of Earth and Environment, Boston University, Boston, USA 
scale (SGS) turbulence closure (or a SGS model). The fidelity of the LES technique depends on the SGS model, the numerical schemes, and the grid spacing (Pope 2000). A standard procedure for researchers using the LES approach is to set up sensitivity experiments with different grid spacings in order to ensure that the chosen grid spacing is fine enough to resolve the relevant scales of the turbulent flow in question. Only when the flow features to be studied do not change when further decreasing the grid spacing is a suitable grid spacing achieved. Hereafter we refer to the sensitivity of LES results to the grid spacing as the grid-sensitivity issue or the lack of grid convergence.

While no major grid-sensitivity issue has been reported for simulating convective and neutral boundary layers in the LES literature, the first intercomparison study of LES of the (weakly) stable boundary layer (SBL) in the scope of the Global Energy and Water Cycle Experiment Atmospheric Boundary Layer Study 1 (GABLS1, Beare et al. 2006) revealed an unsatisfying lack of grid convergence. In particular, it was found that the boundary-layer height decreased significantly with decreasing grid spacing. This key finding was confirmed and cited in numerous other LES studies, which used much smaller grid spacings than those in the original GABLS1 set-up (e.g., Fernando and Weil 2010; van Stratrum and Stevens 2015; Sullivan et al. 2016; Maronga and Bosveld 2017). Maronga et al. (2020b) hypothesized that this grid sensitivity in stable conditions is related to flaws in the surface boundary conditions used in all contemporary LES models. To test that hypothesis they implemented a corrected boundary condition. Their results showed, however, that the observed grid sensitivity was not caused by the previously found grid dependence of the surface fluxes and thus disproved this hypothesis. Recently, Dai et al. (2021) concluded from their LES runs of the GABLS1 case that the lack of grid convergence is caused by the mixing-length formulation in the Deardorff SGS model (Deardorff 1980, hereafter referred to as the D80 model), which is a widely used SGS model, especially for atmospheric applications. In the Deardorff SGS model, the SGS turbulent fluxes are parametrized through flux-gradient relations and the SGS eddy viscosity and diffusivities are linked to the SGS turbulence kinetic energy (TKE). Dai et al. (2021) argued that the parameterization of the original SGS mixing length is flawed for small grid spacings, and they proposed a correction that leads to much improved grid convergence, at least for the range of grid spacings between $12.5 \mathrm{~m}$ and $2 \mathrm{~m}$ and for the GABLS1 case. More specifically, their reasoning is based on the equation to calculate the SGS mixing length $l$, viz.

$$
l= \begin{cases}\min \left(1.8 z, \Delta, L_{\mathrm{b}}\right) & \text { for } \frac{\partial \theta_{\mathrm{v}}}{\partial z}>0 \\ \min (1.8 z, \Delta) & \text { for } \frac{\partial \theta_{\mathrm{v}}}{\partial z} \leq 0\end{cases}
$$

where $z$ is the height above ground, $\Delta$ is the grid spacing, $\theta_{\mathrm{v}}$ is the virtual potential temperature, and $L_{\mathrm{b}}$ is a buoyancy scale, defined as

$$
L_{\mathrm{b}}=\frac{c_{\mathrm{b}} \sqrt{e}}{N}
$$

Here, $c_{\mathrm{b}}$ is a constant, $e$ is the mean TKE, and $N$ is the Brunt-Väisälä frequency. This formulation has its origin in the Deardorff SGS model and was designed to limit $l$ in the case of $L_{\mathrm{b}}<\Delta$. Note that the original Deardorff SGS model did not consider the distance to the wall as a limiting factor, but in many LES models, such as the PALM model used here, the wall effect is often implicitly included (e.g., through the 1.8z term in Eq. 1). Dai et al. (2021) discussed that for today's LES grid spacings that are on the order of $1-10 \mathrm{~m}, L_{\mathrm{b}}$ is usually larger than $\Delta$ even under stable conditions. As a direct consequence, the minimum operation 
in Eq. 1 would not work as designed and $l$ is always equal to $\Delta$ (except near the wall where the $1.8 z$ term comes into play). This implies that stratification is not felt by the SGS model in such cases, which seems non-physical. However, one might argue that this behaviour is plausible, since the SGS model deals only with the SGS turbulence, which should not feel the stable stratification if the grid spacing is very small. Moreover, while their alternative formulation for $l$,

$$
\frac{1}{l}=\frac{1}{\kappa z}+\frac{1}{L_{\mathrm{b}}}
$$

where $\kappa=0.4$ is the von Kármán constant and $\kappa z$ represents the wall effect, results in much better grid convergence, it can easily lead to $l>\Delta$. This behaviour might be put into question by the reasoning that the size of the SGS eddies ( $l$ can be seen as a measure for their diameter) cannot be larger than the grid spacing.

Closer inspection of the data of Dai et al. (2021) reveals that, although grid sensitivity appears to have vanished at grid spacings down to $3.125 \mathrm{~m}$, it actually reappears when the grid spacing goes further down to $1 \mathrm{~m}$ (see their Fig. 1, right panel). This was hidden by the multitude of lines in their plots and thus was not properly discussed. We revisit some of their simulations and based on additional experiments we report that there is a substantial, but still partly limited, grid convergence for the proposed modification by Dai et al. (2021). The grid sensitivity was also shown to persist in the mean profiles of temperature and wind speed down to grid spacings of $0.39 \mathrm{~m}$ by Sullivan et al. (2016), though they reported that the average wind shear and buoyancy frequency almost converge between grid spacings of $0.79 \mathrm{~m}$ and $0.39 \mathrm{~m}$. Some authors, however, reported little or no grid sensitivity in their LES results based on the GABLS1 case. Basu and Porte-Agel (2006), for example, used a scaledependent dynamic SGS model and reported no significant differences in bulk boundary-layer parameters based on LES runs with relatively coarse grid spacings between $12.5 \mathrm{~m}$ and $5 \mathrm{~m}$. Both Stoll and Porte-Agel (2008) and Lu and Porte-Agel (2013) reported little grid sensitivity using a nonlinear SGS flux model and a modulated gradient SGS model, respectively. Huang and Bou-Zeid (2013) stated that their simulations of the GABLS1 case have reached grid convergence between grid spacings of $3.125 \mathrm{~m}$ and $2.5 \mathrm{~m}$. Van der Linden et al. (2019) used very fine grid spacings of $0.125 \mathrm{~m}$ and $0.08 \mathrm{~m}$ in their LES of a very stable boundary layer and reported no severe grid sensitivity. However, on close inspection of all mentioned studies, their data display noticeable effects of the grid spacing down to the smallest grid spacing tested. Above all, a decrease in the boundary-layer height can be identified in all studies. The data shown by Huang and Bou-Zeid (2013), for instance, reveal differences in the mean and flux profiles between the two smallest employed grid spacings (their Fig. 1). Their conclusion was probably reasonable since the reported differences were on the order of only 3-4\%, but one could observe a slightly shallower boundary layer and smaller vertical fluxes for the smaller grid spacing (2.5 m). Van der Linden et al. (2019) reported no severe grid sensitivity, though their data show the same tendency towards lower boundary-layer heights with decreasing grid spacing (see their Fig. 9). We thus conclude that the issue of grid sensitivity persists, though the effect was sometimes reported to be rather small. Note that so far no objective measure has been proposed to define grid convergence, so that statements about grid sensitivity have been somewhat arbitrary.

Beyond these studies, we are not aware of other work that specifically investigates the causes of the grid-sensitivity issue when simulating the SBL. Hence we can only speculate on the possible reasons, starting with deficiencies of the SGS models, effects of entrainment or mixing with the free atmosphere above, feedback between the near-surface flow and temperature gradients and the surface boundary conditions, issues with the application of 
Monin-Obukhov similarity theory (MOST, Monin and Obukhov 1954) in LES models (as suggested by Maronga et al. 2020b), dissipation and dispersion of the numerical schemes, or generally under-resolved turbulent flow.

In the present paper we make use of a comprehensive set of LES of the GABLS1 case to demonstrate that none of the above-mentioned processes or factors seem to fully explain and resolve the grid-sensitivity issue. In Sect. 2, we describe the experimental set-up of the LES runs. Results are presented and discussed in Sect. 3, and Sect 4 presents a summary.

\section{Experimental Set-up}

\subsection{The Large-Eddy Simulation Model}

The LES model PALM in revision 4508 is used to solve the equations of conservation for momentum, heat, and moisture in the Boussinesq-approximated form on a Cartesian staggered Arakawa-C grid. It has been widely used to study convective boundary layers (e.g., Raasch and Franke 2011; Maronga et al. 2015), as well as turbulent flows under neutral (e.g. Knigge et al. 2015; Maronga et al. 2020b) and stable conditions (e.g., Beare et al. 2006; Maronga and Bosveld 2017; Maronga et al. 2020b). Maronga et al. (2015, 2020a) provide more details on PALM.

The simulations were carried out using cyclic lateral boundary conditions. Discretization in time was achieved by a third-order Runge-Kutta time-stepping scheme (Williamson 1980). We used several schemes for discretization in space: a fifth-order advection scheme (Wicker and Skamarock 2002) (hereafter referred to as the WS02 scheme), which is a widely-used scheme in LES models. The advantage of the WS02 scheme is its small dispersive error so that small-scale turbulent structures are well maintained. Nonetheless, it suffers from immense numerical energy dissipation at high wavenumbers (small scales). As a consequence, the spectral energy density decreases rapidly at high wavenumbers. For comparison, we performed runs with the second-order scheme proposed by Piacsek and Williams (1970) (hereafter referred to as the PW70 scheme), which is non-dissipative but has large dispersive errors. As a consequence, small-scale turbulence structures are not well-preserved when using the PW70 scheme. Finally, we also conducted experiments with a monotonic, positive definite scheme for temperature (Chlond 1994, hereafter the BC94 scheme after the key authors Bott and Chlond), with dynamic quantities still treated by the WS02 scheme. The BC94 scheme has the advantage of better resolving local scalar gradients, which is potentially beneficial for simulating mixing with the free atmosphere, but it is much more computationally expensive. Note that the BC94 scheme also has a significant amount of numerical dissipation. See the Appendix for an illustration of the effect of model numerics on turbulence spectra.

In terms of SGS model, PALM uses the D80 turbulence closure in the default configuration. For some runs we also used a revised D80 model proposed by Dai et al. (2021) (hereafter referred to as the D80-R model). Moreover, we used implicit LES (ILES, see, e.g., Smolarkiewicz et al. 2007; Adams and Hickel 2009), where the SGS model is switched off and where the required dissipation is only provided by the numerical schemes.

For most runs, MOST was invoked to calculate surface sensible heat and momentum fluxes as boundary conditions. In order to exclude feedback between the mean wind speed and temperature profiles in the near-surface region and the surface sensible heat and momentum fluxes, we conducted additional runs in which we fixed the horizontally-averaged friction velocity $\left(u_{*}\right)$ and MOST temperature scale $\left(\theta_{*}\right)$. 
Table 1 Overview of LES runs

\begin{tabular}{|c|c|c|c|c|c|c|c|c|c|}
\hline \multirow[t]{2}{*}{ Set } & \multicolumn{9}{|l|}{$\Delta$} \\
\hline & $12.5 \mathrm{~m}$ & $10 \mathrm{~m}$ & $8 \mathrm{~m}$ & $6.25 \mathrm{~m}$ & $5 \mathrm{~m}$ & $4 \mathrm{~m}$ & $3.125 \mathrm{~m}$ & $2 \mathrm{~m}$ & $1 \mathrm{~m}$ \\
\hline D80 & $\mathrm{x}$ & $\mathrm{x}$ & $\mathrm{x}$ & $\mathrm{x}$ & $\mathrm{x}$ & $\mathrm{x}$ & $\mathrm{x}$ & $\mathrm{x}$ & $\mathrm{x}$ \\
\hline D80-R & $\mathrm{x}$ & $\mathrm{x}$ & $\mathrm{x}$ & $\mathrm{x}$ & $\mathrm{x}$ & $\mathrm{x}$ & $\mathrm{x}$ & $\mathrm{x}$ & $\mathrm{x}$ \\
\hline D80_PW70 & $\mathrm{x}$ & $\mathrm{x}$ & $\mathrm{x}$ & $\mathrm{x}$ & $\mathrm{x}$ & $\mathrm{x}$ & $\mathrm{x}$ & $\mathrm{x}$ & - \\
\hline D80-R_PW70 & $\mathrm{x}$ & $\mathrm{x}$ & $\mathrm{x}$ & $\mathrm{x}$ & $\mathrm{x}$ & $\mathrm{x}$ & $\mathrm{x}$ & $\mathrm{x}$ & - \\
\hline ILES & $\mathrm{x}$ & $\mathrm{x}$ & $\mathrm{x}$ & $\mathrm{x}$ & $\mathrm{x}$ & $\mathrm{x}$ & $\mathrm{x}$ & $\mathrm{x}$ & $\mathrm{x}$ \\
\hline ILES_BC94 & $\mathrm{x}$ & $\mathrm{x}$ & $\mathrm{x}$ & $\mathrm{x}$ & $\mathrm{x}$ & $\mathrm{x}$ & $\mathrm{x}$ & $\mathrm{x}$ & - \\
\hline ILES_BC94_FIX & $\mathrm{x}$ & $\mathrm{x}$ & $\mathrm{x}$ & $\mathrm{x}$ & $\mathrm{x}$ & $\mathrm{x}$ & $\mathrm{x}$ & $\mathrm{x}$ & - \\
\hline
\end{tabular}

\subsection{Case Description and Model Set-up}

We simulated the GABLS1 case, which is a weakly SBL driven by a geostrophic wind speed of $8 \mathrm{~m} \mathrm{~s}^{-1}$ and geostrophic direction from the west, and an imposed surface cooling rate of $0.25 \mathrm{~K} \mathrm{~h}^{-1}$. The domain size was roughly $400 \times 400 \times 400 \mathrm{~m}^{3}$. A roughness length of $0.1 \mathrm{~m}$ was used for both momentum and heat. The initial potential temperature $(\theta)$ was set to a constant value of $265 \mathrm{~K}$ up to $100 \mathrm{~m}$, with an overlying inversion of $0.01 \mathrm{~K} \mathrm{~m}^{-1}$. In order to trigger turbulence, random temperature perturbations were imposed on the initial temperature field. The latitude was set to $73^{\circ} \mathrm{N}$, which corresponds to a Coriolis parameter of $1.39 \times 10^{-4} \mathrm{~s}^{-1}$. The atmosphere was considered to be dry, i.e., no equation for moisture was solved. As with Dai et al. (2021), we performed runs with a wide range of isotropic grid spacings between $12.5 \mathrm{~m}$ and $1 \mathrm{~m}$.

Overall, we ran six sets of LES (59 simulations in total) with different model configuration. Table 1 gives an overview of these LES runs. As in Dai et al. (2021), we ran two sets of simulations with the D80 and D80-R models (but with an extended simulation period, see below) in combination with the WS02 scheme (sets D80 and D80-R). Additionally, we performed two sets of simulations where we replaced the WS02 scheme with the PW70 scheme in order to study the effects of numerical dissipation and physical dissipation by the SGS models (sets D80_PW70 and D80-R_PW70). In order to investigate whether the gridsensitivity issue is related to the SGS model, we ran a full set of simulations in the ILES mode (i.e., without any SGS model). Based on the results (shown and discussed below) we decided to use the ILES set-up together with the BC94 advection scheme for scalar quantities to better represent vertical gradients of temperature near the surface and the top of the boundary layer (set ILES_BC94). In the last set, we further fixed the surface fluxes of momentum and sensible heat by prescribing the mean (horizontal average) friction velocity and MOST temperature scale in order to examine whether the grid sensitivity is caused by the surface boundary condition or its interplay with the near-surface resolved flow (set ILES_BC94_FIX). Note that we could only run the cases D80, D80-R, and ILES with the finest grid spacing of $1 \mathrm{~m}$ due to the high computational demands of such simulations.

\subsection{Data Processing}

In the original GABLS1 model intercomparison study (Beare et al. 2006), simulations were performed over $9 \mathrm{~h}$ and data were averaged and compared for the last simulated hour. During 


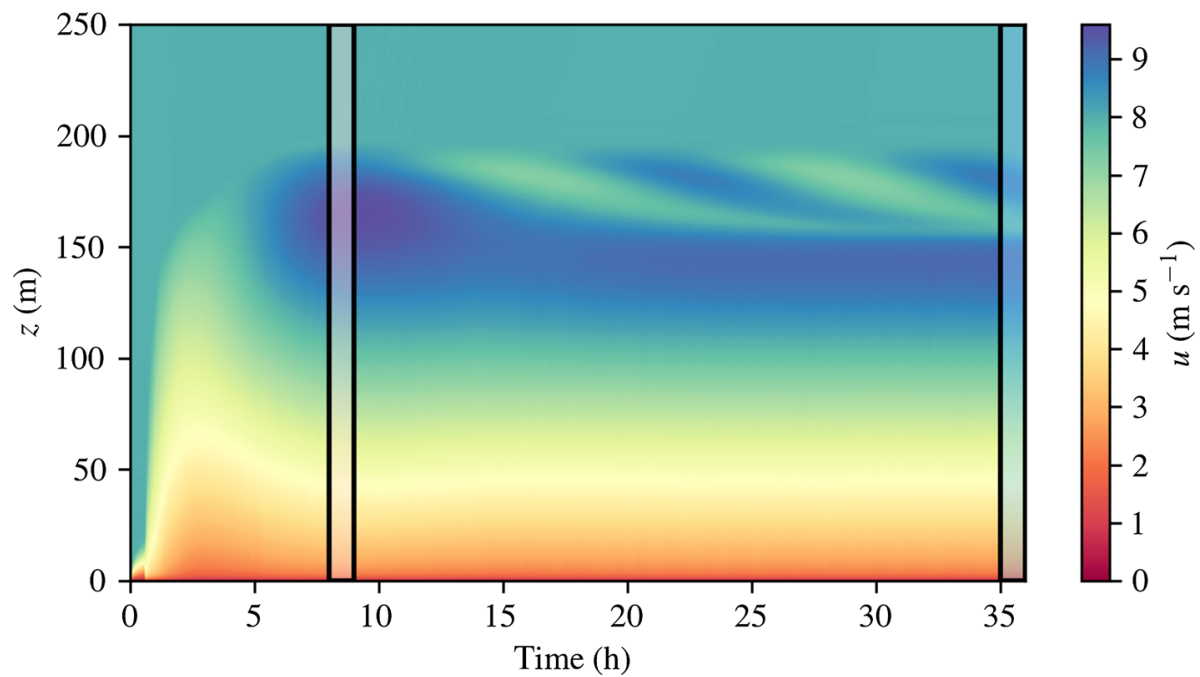

Fig. 1 Time-height cross-section of the horizontally-averaged $u$ velocity component for case D80 for a grid spacing of $1 \mathrm{~m}$. Reddish semi-transparent areas denote the averaging interval used by Beare et al. (2006) and Dai et al. (2021), while the blueish semi-transparent areas show the averaging interval employed here

the analysis of the LES data here, we were able to reproduce the original GABLS1 results when we used a simulation period of $9 \mathrm{~h}$. However, when we extended the simulation time we found that the mean state of the boundary layer was still evolving after $9 \mathrm{~h}$. In particular, the flow was clearly affected by the development of an inertial oscillation, which is illustrated in Fig. 1. The inertial oscillation developed after about $12.5 \mathrm{~h}$ in the simulations. This time scale is imposed by the Coriolis force as $\tau_{i o}=2 \pi / f$, where $f$ is the Coriolis parameter of $1.39 \times 10^{-4} \mathrm{~s}^{-1}$ (corresponding to a latitude of $73^{\circ} \mathrm{N}$, see above). Hence, the shorter simulation period used in all previous LES studies of the GABLS1 case may not be adequate to reach steady-state conditions.

As a direct consequence, we extended the simulation time for all runs to $36 \mathrm{~h}$, which covers at least three full periods of the inertial oscillation. Vertical profiles were then horizontally and temporally averaged over $1 \mathrm{~h}$ of simulation time from hours 35-36. Note that for the wind speed profiles, longer time-averaging periods (e.g., over a period of $n \tau_{i o}$ where $n$ is a positive integer) could be considered to cancel out the effects of the inertial oscillation (Momen and Bou-Zeid 2017). However, as the temperature continues to drop, using longer averaging periods would result in substantial changes in the thermodynamic quantities. In order to maintain some consistency with previous studies for comparison purposes, we decided to use 1-h averages.

Furthermore, Dai et al. (2021) found that the onset of turbulence for the D80 cases displayed a strong grid-size sensitivity. The present study confirms this finding: the coarser the grid spacing, the later the onset of turbulence. This is indicated by the sudden spikes in the time series of TKE that differ by up to several hours (see Fig. 2a). Except the D80-R case, all others show this sensitivity. We can ascribe this striking feature to the fact that the D80 model produces a much larger eddy diffusivity near the surface than the D80-R model (Dai et al. 2021). As turbulence is triggered by initial temperature perturbations close to the surface, which creates unrealistically high second derivatives, the SGS diffusion leads to continuous destruction of turbulent fluctuations, which effectively delays the development of turbulence 
(see also Donda et al. 2015). This effect is amplified with increasing grid spacings. In fact, Dai et al. (2021) showed that for very coarse grid spacings, no turbulence is developed at all when using the D80 model. Here, we strictly followed the GABLS1 set-up and used a single temperature perturbation at model start. There can be other ways of initializing turbulence. In fact, by default PALM uses repeated perturbations of the velocity field instead, which might overcome the issue of large differences in the onset time of turbulence. Alternatively, a more realistic initial turbulence field could be used to avoid rapid dissipation of the perturbations. These methods are not tested here and are left for future research.

While this phenomenon was shown by Dai et al. (2021), they did not discuss its direct consequence on the comparability of mean profiles for runs with different grid spacings. As the SBL is continuously cooled by a prescribed decreasing surface temperature, a true steady-state does not exist for the temperature profile (see, e.g., Derbyshire 1990). The surface heat flux is calculated interactively via MOST. The calculated surface heat flux is directly affected by turbulent mixing (of lack thereof) and strongly depends on whether turbulence has developed or not. The energy removed from the system by cooling from the surface is thus not identical between runs. This becomes a concern if the onset of turbulence is vastly different and if the simulation time is short since the energy in the system would then vary strongly between runs, which renders the comparison of vertical profiles - at least for temperature-problematic. Indeed, we found the non-monotonous change of the mean temperature profiles with increasing grid spacing, as shown by Dai et al. (2021), to be an artefact of this incomparable condition. By extending the simulation time to $36 \mathrm{~h}$, differences in the cooling magnitude during the first hours of the simulations (i.e., during spin-up) become eventually negligible compared to the total energy removed from the system by the negative surface heat flux, allowing thermodynamic quantities to be compared. While this effect does heavily influence the mean temperature profiles, the mean wind profiles show less a response for which a steady-state can be reached. Note that improved model initialization as discussed above could potentially remove the issue of de-synchronization of the runs.

\section{Results}

\subsection{Mean Profiles}

Vertical profiles of mean potential temperature and wind speed $\left(u_{\mathrm{h}}\right)$ for cases D80 and D80$\mathrm{R}$ are shown in Fig. 3 ( $\mathrm{a}-\mathrm{b}$, e-f). In general, we observe negligible differences among the two cases with different grid spacings in the lower part of the boundary layer. For the D80 cases, both temperature and wind-speed profiles reveal increasing boundary-layer heights with decreasing grid spacings until $\Delta=3.125 \mathrm{~m}$. This is in contrast with the results shown by Dai et al. (2021) and can be explained by the different time periods used for analysis (i.e., $9 \mathrm{~h}$ vs. $36 \mathrm{~h}$, see reasoning above). However, with smaller grid spacings of $2 \mathrm{~m}$ and $1 \mathrm{~m}$, we observe decreasing boundary-layer heights. This is consistent with the intercomparison results for GABLS1 as discussed by Beare et al. (2006). We might ascribe this partly to the fact that the onset times of turbulence are better synchronized (cf. Fig. 2). Nevertheless, the same grid sensitivity for fine grid spacings $(2 \mathrm{~m}$ and $1 \mathrm{~m}$ ) remains visible for the D80-R model, though it is considerably weaker. We also observe that the D80 runs yield greater boundary-layer heights than the D80-R runs with coarse grid spacings ( $\Delta \geq 3.125 \mathrm{~m}$ ), while both D80 and D80-R predict surprisingly similar boundary-layer heights with the finest grid spacing $(\Delta=1.0 \mathrm{~m})$. This is further compared and discussed later. 

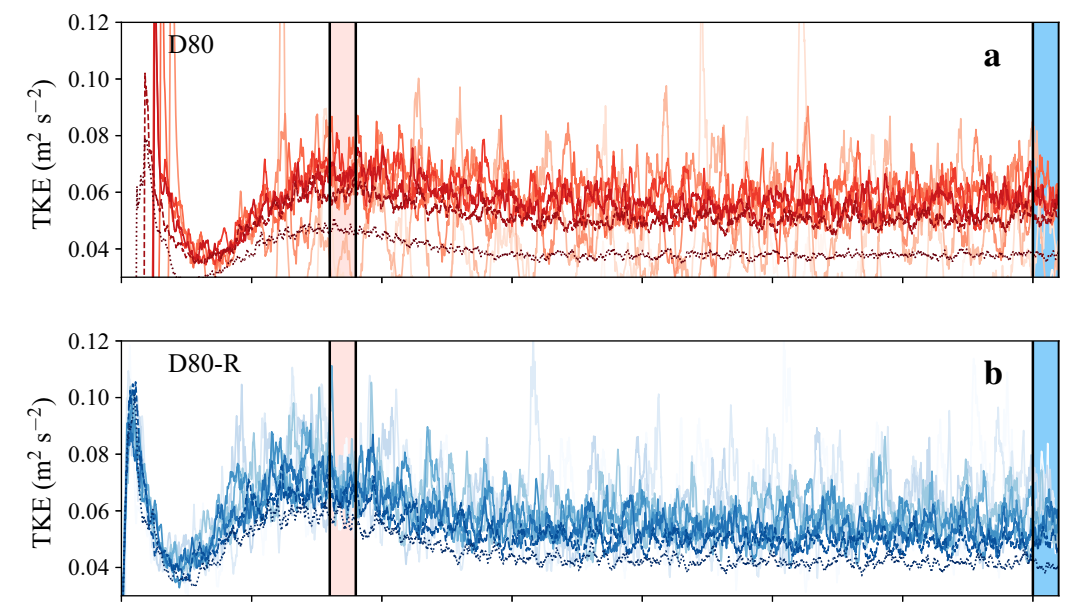

$-10$

8

6.25

5
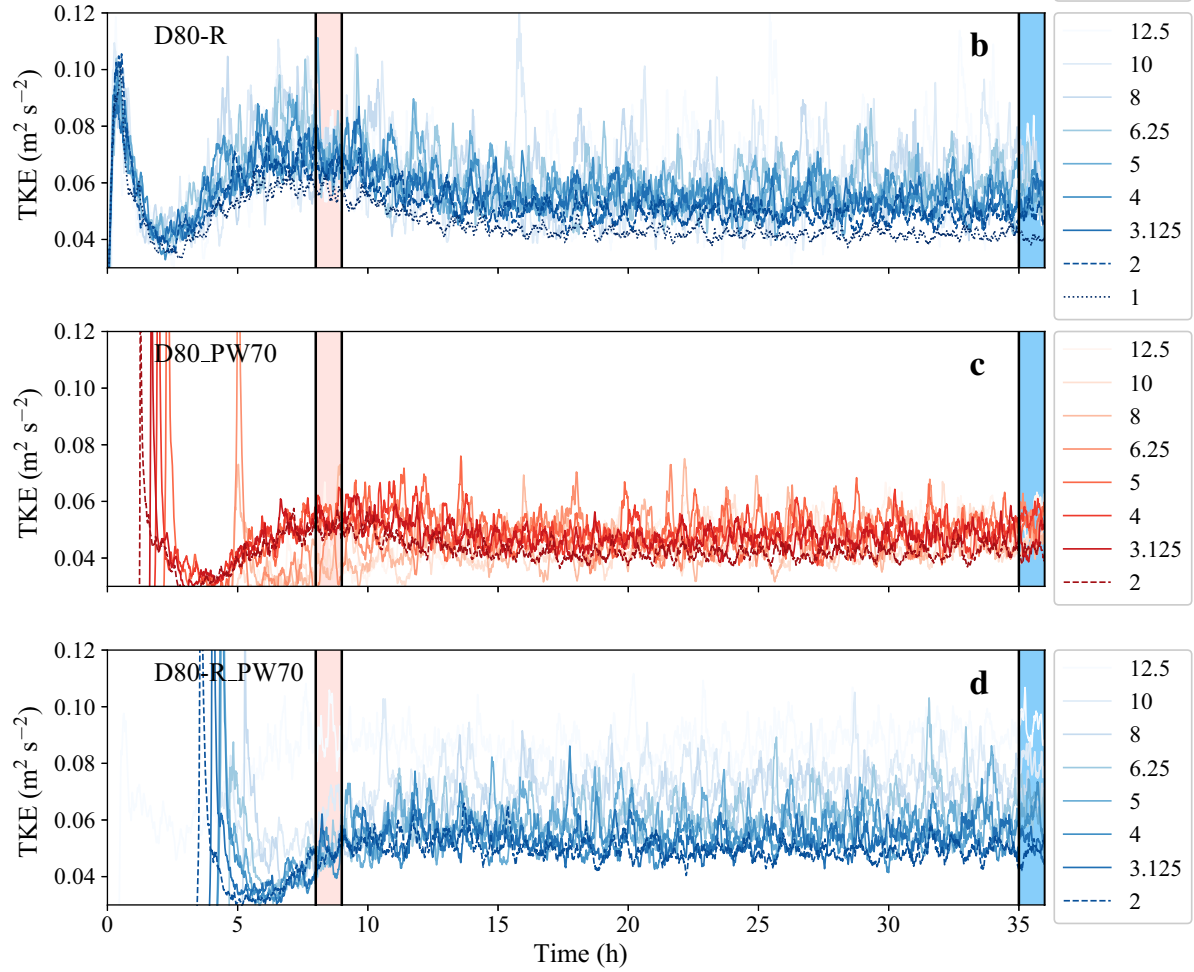

Fig. 2 Time series of the domain-averaged TKE for cases D80 (a), D80-R (b), D80_PW70 (c), and D80R_PW70 (d). Red-shaded areas denote the averaging interval that was used by Beare et al. (2006) and Dai et al. (2021), while the blue-shaded areas show the averaging interval employed

The wind-speed profiles shown in Fig. 3 e-f show consistent features with the temperature profiles and thus are not discussed in detail. Note, however, that in all runs we observe a secondary wind speed maximum above the boundary layer, which was neither found by Beare et al. (2006) nor by Dai et al. (2021). This secondary wind-speed maximum is related to the inertial oscillations discussed in Sect. 2.3 (Fig. 1). As these oscillations do not alter the temperature profiles, they are only visible in the wind-speed profiles. Because of these oscillations, the wind speed and direction above the boundary layer vary in time. Again, the simulation time of only $9 \mathrm{~h}$ used in previous studies is too short and the inertial oscillations had not developed (cf. Fig. 1). 

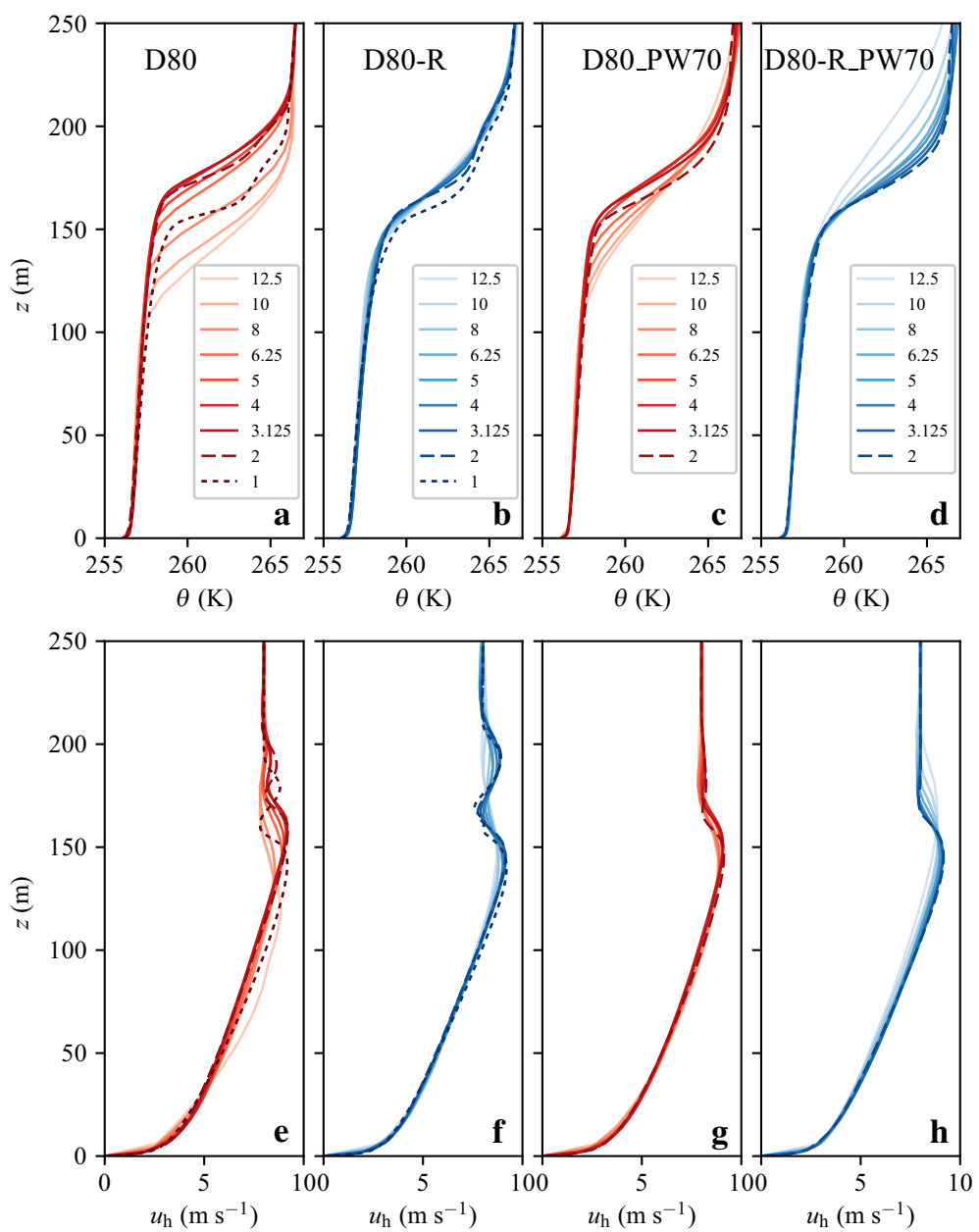

Fig. 3 Vertical profiles of $\theta(\mathbf{a}-\mathbf{d}), u_{\mathrm{h}}(\mathbf{e}-\mathbf{h})$ for cases D80, D80-R, D80_PW70, and D80-R_PW70, for all grid spacings used. These profiles are time-averaged over the last simulation hour. The colour intensity reflects the grid spacing, so that light colours denote coarse grid spacings while dark colours indicate small grid spacings

\subsection{Turbulent Fluxes}

Vertical profiles of the turbulent heat flux all display linear decreases with height (Fig. 4 $\mathrm{a}-\mathrm{b}, \mathrm{c}-\mathrm{d}$ ), except for the coarsest grid spacing in the D80 set, where turbulence was not fully developed. The grid-size dependence is very similar to that for the mean profiles of temperature and wind speed as discussed before. For both D80 and D80-R sets, the heat-flux profiles approach quasi-converged states down to a grid spacing of $3.125 \mathrm{~m}$. For even smaller grid spacings (i.e., $2 \mathrm{~m}$ and $1 \mathrm{~m}$ ), the lower boundary-layer heights shown in Fig. 3 are clearly associated with lower heat fluxes (in the absolute sense) and lower Reynolds stresses near the surface shown in Fig. 4. This is again more pronounced for the D80 runs than the D80-R runs. 

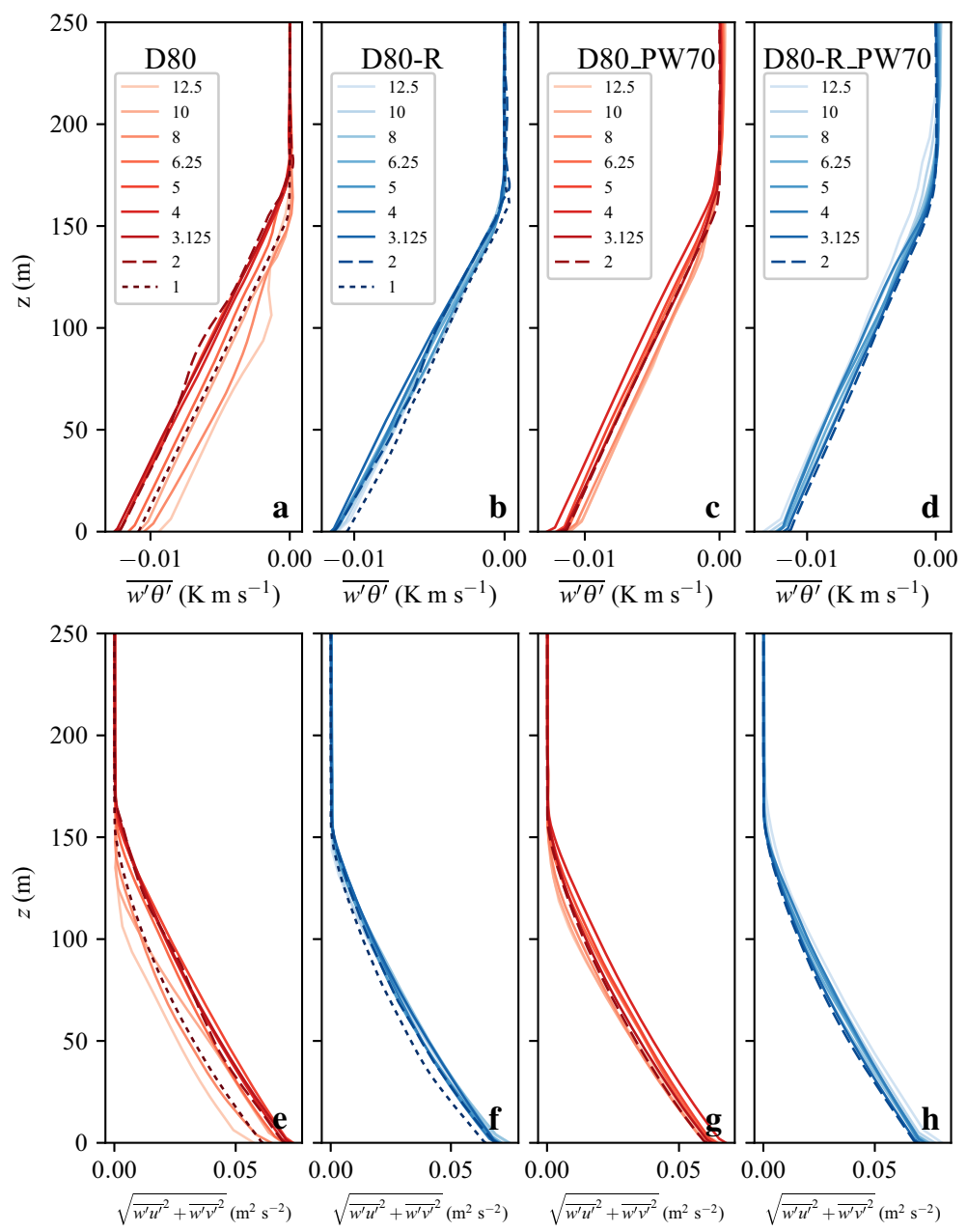

Fig. 4 As Fig. 3 but for the turbulent heat flux $\overline{w^{\prime} \theta^{\prime}}$ (a-d) and the Reynolds stress $\left({\overline{w^{\prime} u^{\prime}}}^{2}+{\overline{w^{\prime} v^{\prime}}}^{2}\right)^{1 / 2}(\mathbf{e}-\mathbf{h})$

\subsection{Turbulence Kinetic Energy}

The analysis of the mean profiles and turbulent fluxes clearly indicates that for both D80 and D80-R runs, grid convergence is found down to a grid size of about $3.125 \mathrm{~m}$, but that grid sensitivity arises again when further decreasing the grid spacing to $2 \mathrm{~m}$ and $1 \mathrm{~m}$. This finding is consistent with the domain-averaged TKE shown in Fig. 2a, b. Here we observe a decrease in TKE when the grid spacing changes from $3.125 \mathrm{~m}$ to $2 \mathrm{~m}$ and $1 \mathrm{~m}$, which is clearly visible for both the D80 and D80-R cases. Dai et al. (2021) did not report this sensitivity, possibly because it is only weakly developed when using the D80-R model at $9 \mathrm{~h}$. However, our results clearly demonstrate that the grid sensitivity still exists and that there is no true convergence at grid spacings $>1 \mathrm{~m}$. Hence the D80-R model largely improves but does not fully resolve the grid-sensitivity issue. 


\subsection{Analysis of Possible Causes of Grid Sensitivity}

As we outlined in Sect. 2, we performed additional LES experiments to investigate several possible causes of the grid sensitivity. In the following we discuss the results from these simulations.

\subsubsection{Numerical Dissipation}

The baseline runs (i.e., D80 and D80-R) were conducted with the WS02 advection scheme, which is highly dissipative (Wicker and Skamarock 2002). Based on our previous experience the scheme also has issues with the treatment of vertical gradients, which typically manifest in the entrainment zone or the inversion layer. Figure $3 \mathrm{a}, \mathrm{b}$ illustrate this feature. One can see that the temperature gradient changes rather abruptly in the $1 \mathrm{~m}$-run at heights of about $160 \mathrm{~m}$ and $180 \mathrm{~m}$, separating the inversion into several layers with different static stability. In order to study how these properties of the WS02 scheme might affect the grid-sensitivity issue, we performed additional runs with the PW70 scheme (cases D80_PW70 and D80-R_PW70), which does not suffer from numerical dissipation. Dissipation of TKE is thus accomplished entirely by the SGS models for these cases. Note that the PW70 scheme, while being nondissipative, has large dispersive errors so that the spatial coherence of turbulent structures is not well preserved and thus is generally inferior to the WS02 scheme.

In Fig. 3c, d, vertical profiles of mean potential temperature are shown. Above all, we note a significant grid sensitivity for both the D80_PW70 and the D80-R_PW70 cases, and a completely different behaviour in comparison to the baseline D80 and D80-R runs (cf. Fig. 3a, b). For the D80_PW70 we find that the grid sensitivity is reduced compared to D80, while for D80-R_PW70 we observe a stronger grid sensitivity compared to D80-R. For D80_PW70 we observe the same decrease of boundary-layer height for grid spacings tending to $2 \mathrm{~m}$ as for D80. For D80-R_PW70 it is striking that the boundary-layer height is monotonously decreasing with decreasing grid spacings, and that no grid convergence is reached at $2 \mathrm{~m}$. Unfortunately, we were unable to run these cases at $1 \mathrm{~m}$ grid spacing because of the extremely high computational demands of the D80-R case. This is because of the relatively large SGS eddy diffusivities calculated by the D80-R model in case of small grid spacings (see Dai et al. 2021, their Fig. 1), which drastically limit the LES timestep. The behaviours of wind-speed profiles (Fig. 3g, h), as well as turbulent flux profiles (see Fig. 4) and the TKE (see Fig. 2), are in line with the temperature profiles. For D80-R_PW70 we also note a significantly delayed onset of turbulence and hence a low TKE during the first $4 \mathrm{~h}$. For the wind-speed profiles, we note that the inertial oscillations above the boundary layer are only weakly developed with the PW70 scheme, which we ascribe to the large dispersive errors of the PW70 scheme.

Based on this analysis, we can conclude that numerical dissipation is a key factor that affects how the grid sensitivity is manifested, and it interacts with the SGS model. As the mean profiles vary strongly between runs with the WS02 and PW70 schemes (see Fig. 3), it can be only concluded that the grid-sensitivity issue cannot be fully resolved by switching to a vastly different numerical scheme.

\subsubsection{Sub-grid Scale Model}

Motivated by the finding that the D80-R model does not fully resolve the grid-sensitivity issue, and the observation that the boundary-layer height for all cases (i.e., D80, D80-R, 
D80_PW70, D80-R_PW70) approaches similar values when $\Delta \leq 2 \mathrm{~m}$ (see Fig. 5), we performed a set of runs in the ILES mode (ILES cases), i.e., without any SGS model. In the ILES cases, the dissipation of energy is solely achieved by the numerical scheme (i.e., the WS02 scheme).

Figure 5a, $d$ shows the mean profiles for the ILES cases. One can see that the grid sensitivity is less pronounced, but that the general behaviour of the boundary-layer height is the same as in the D80 cases (cf. Fig. 3a, e). That is, the simulations display increasing boundarylayer heights with decreasing grid spacings, converging at grid spacings close to $3.125 \mathrm{~m}$. However, as the grid spacing becomes smaller than $3.125 \mathrm{~m}$, shallower boundary layers are again observed. The former finding can be attributed to the fact that the onset time of turbulence is almost identical for all grid spacings (see Fig. 6). We can explain this by the lack of dissipation by the SGS model, allowing turbulence to develop quickly as a result of the initial perturbations. The latter finding proves that the observed grid sensitivity when the grid spacing becomes smaller than $3.125 \mathrm{~m}$ is likely not caused by deficiencies of the SGS model. The flux profiles do not reveal anything unexpected and are not discussed here.

Figure 7 provides an overview of the mean profiles of D80, D80-R, and ILES cases for the finest grid spacings of 1-2 $\mathrm{m}$. While we clearly see that for $\Delta=2 \mathrm{~m}$ the three cases give differing mean profiles and boundary-layer heights (with the D80-R yielding a boundary layer that is about $25 \mathrm{~m}$ shallower than D80 and ILES), it is striking that for $\Delta=1 \mathrm{~m}$ all three cases reveal very similar mean profiles with differences in boundary-layer height of less than $10 \mathrm{~m}$, which would be suggestive of reaching grid convergence. Here, case D80-R tends to have higher boundary layers and weaker capping inversions than the other two.

Figure 6a again reveals that the runs with fine grid spacings $(2 \mathrm{~m}$ and $1 \mathrm{~m})$ are accompanied with lower TKE. This reinforces our previous finding that true convergence might not be reached at grid spacings larger than $1 \mathrm{~m}$. As we do not have data for grid spacings smaller than $1 \mathrm{~m}$, we do not have the means to prove that the simulation results will eventually converge. However, Sullivan et al. (2016) performed LESs for GABLS1 using grid spacings down to $0.39 \mathrm{~m}$ and found that the boundary layer still becomes shallower and shallower. They used a pseudo-spectral code so that the resolution is identical to the grid spacing, while in non-spectral codes like PALM the true resolution is a few (around six) times the grid spacing. Hence, it is conjectured that runs with $\Delta<1 \mathrm{~m}$ might reveal further decreases in TKE and shallower boundary layers.

Nevertheless, the fact that the results become almost independent of the SGS model when the turbulence is sufficiently well resolved is in line with our LES philosophy. That is, it should always be possible to overcome the deficiencies of the SGS model by increasing model resolution. Our data suggest that the SGS model might eventually be dispensable if a small-enough grid spacing is used, with dissipation provided by the numerical schemes. We also tested the ILES mode for simulations of a convective boundary layer and confirmed that mean profiles and turbulence quantities were indeed independent from the SGS model at a sufficiently small grid spacing (not shown). We thus hypothesize that with $\Delta<1 \mathrm{~m}$ the different set-ups (i.e., D80, D80-R, and ILES) all will produce similar mean profiles, no matter if an (or which) SGS model is applied, whereas the grid sensitivity persists.

\subsubsection{Representation of Vertical Gradients of Temperature}

As discussed above, the WS02 scheme shows some deficiencies in capturing vertical gradients. On the other hand, the large dispersive error of the PW70 scheme is not desirable. Hence, we performed an additional set of simulations where the WS02 scheme was replaced by the BC94 scheme for temperature (ILES_BC94). The BC94 scheme provides numerical 

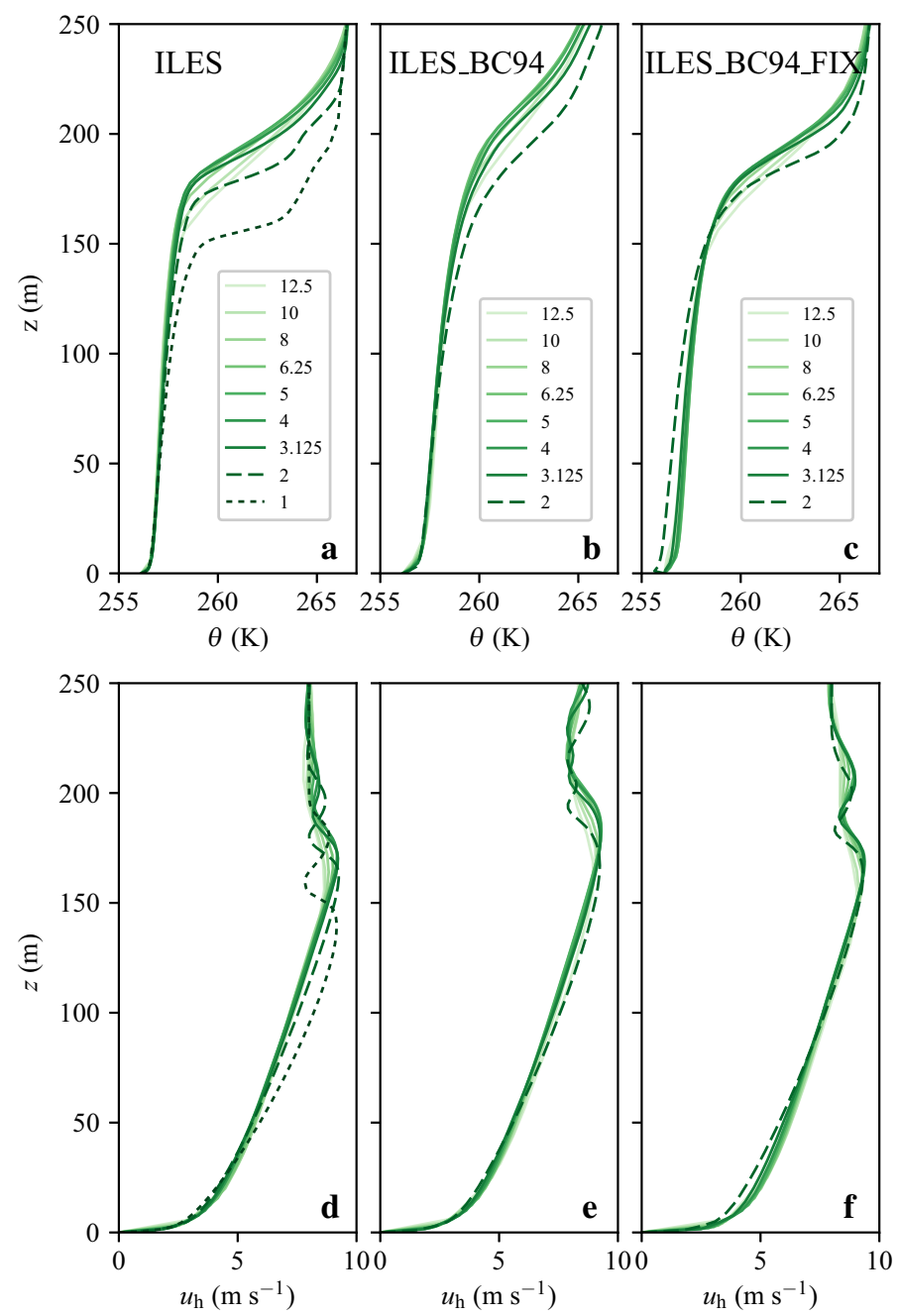

Fig. 5 As Fig. 3 but for cases ILES (a, d), ILES_BC94 (b, e), and ILES_BC94_FIX (c, f)

dissipation, thus being a good option for running in the ILES mode. Note, however, that the BC94 scheme is computationally expensive and hence usually only used for certain applications such as simulating clouds where sharp gradients are important (Chlond 1994; Hoffmann 2017).

Results for case ILES_BC94 are shown in Figs. 5 and 6. While we see distinct differences in the mean profiles compared to the ILES runs (namely, deeper boundary layers and much weaker inversions), the grid sensitivity is not affected. Thus, the representation of vertical temperature gradients both near the surface and near the inversion layer is unlikely the cause of the grid-sensitivity issue. 

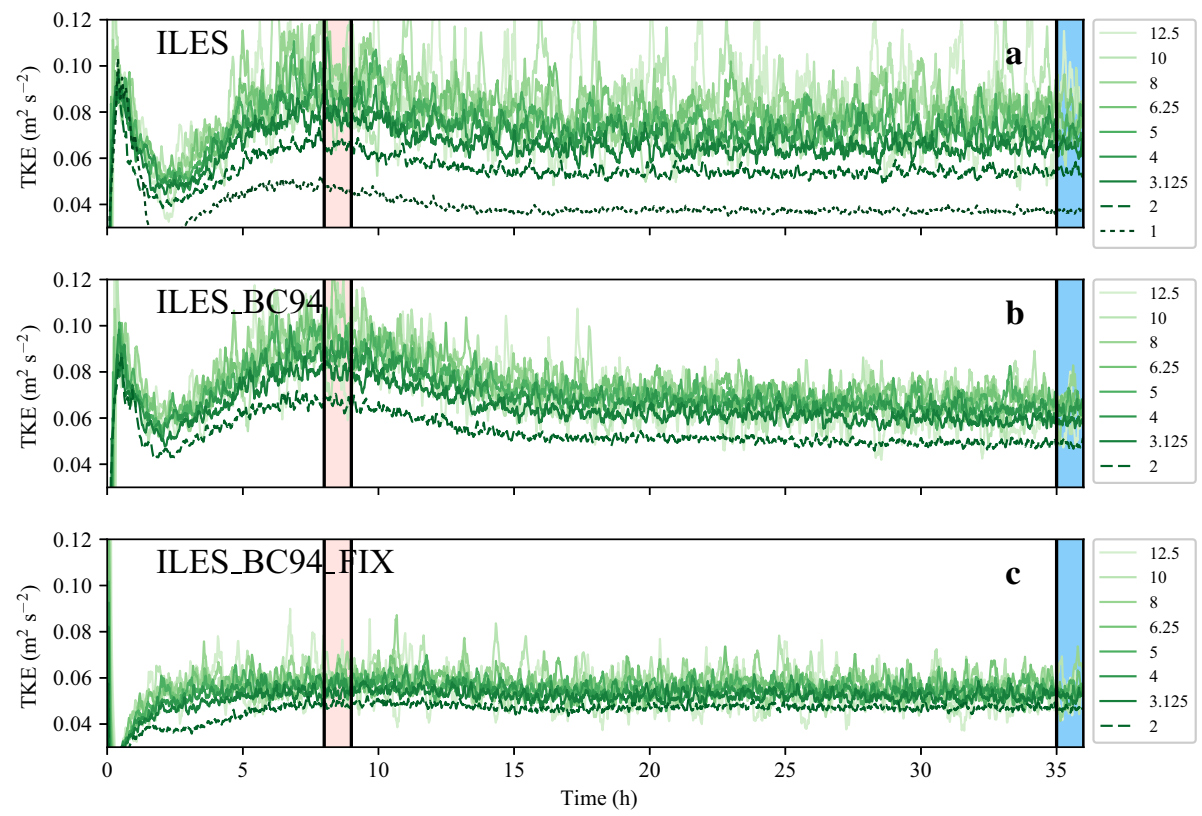

Fig. 6 As Fig. 2 but for cases ILES (a), ILES_BC94 (b), and ILES_BC94_FIX (c)
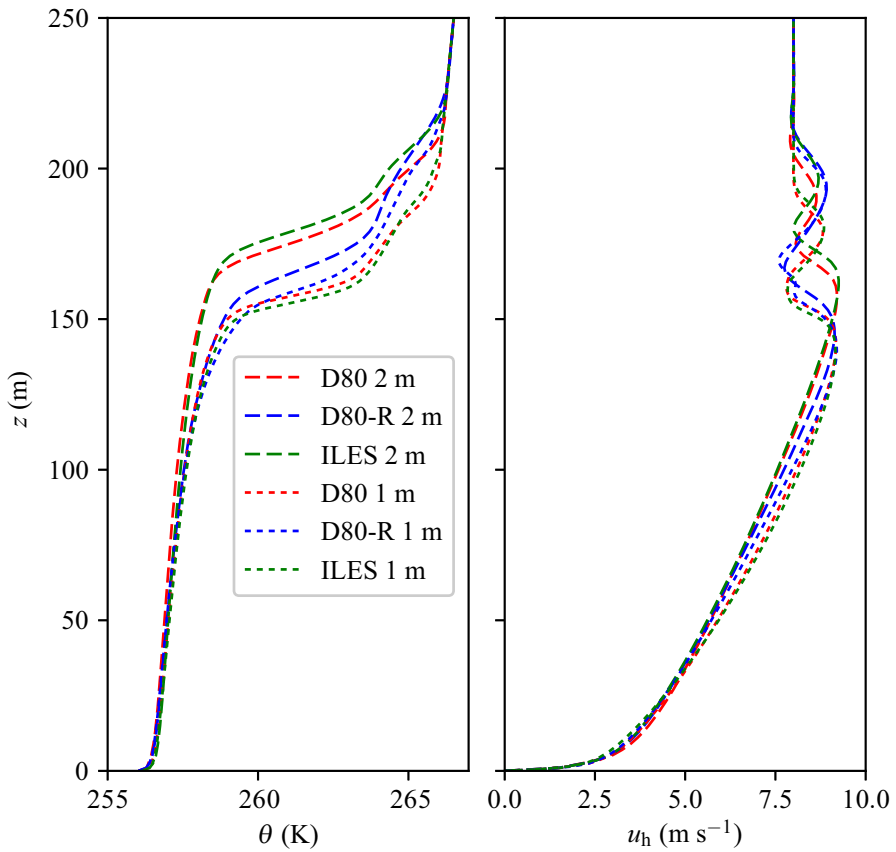

Fig. 7 Vertical profiles of $\theta$ (a) and $u_{\mathrm{h}}$ (b) for cases D80, D80-R, and ILES, for grid spacings of $2 \mathrm{~m}$ and $1 \mathrm{~m}$, time averaged over the last simulation hour 


\subsubsection{Surface Boundary Condition}

Recently, Maronga et al. (2020b) proposed a correction for the interactive calculation of the surface fluxes of heat and momentum via MOST, hypothesizing that the incorrect treatment of the surface boundary condition might be responsible for the grid-convergence issue. Even though they found that their proposed correction did not solve the grid sensitivity, they suggested that other issues of the surface boundary condition, such as inadequate similarity functions for stable conditions or feedback between the surface boundary condition and the (under-resolved) turbulent flow near the surface, should be further examined. In order to follow up on their work and to exclude such effects, we used case ILES_BC94 and fixed the mean surface momentum and heat fluxes, i.e., we prescribed fixed values of the mean $u_{*}$ and $\theta_{*}$ (case ILES_BC94_FIX). As the values of these parameters varied between runs with different grid spacings for the ILES case, we made an ad hoc decision to use the timeaveraged values from the ILES_BC94 $2 \mathrm{~m}$-run. The local surface fluxes were then calculated based on a modulation of the mean $u_{*}$ and $\theta_{*}$ according to the local flow field at the first grid level above the surface to maintain the correlation between the surface and the local flow (for details see Maronga et al. 2020a, b).

Figures 5 and 6 reveal that fixing the surface flux affects both the mean profiles and the TKE. For the TKE we find generally lower values than those in the case ILES_BC94, but the spread between the runs with different grid spacings has reduced significantly. The mean profiles indeed indicate that the grid sensitivity in terms of boundary-layer height has been slightly reduced for runs down to $3.125 \mathrm{~m}$ grid spacing when compared to both ILES and ILES_BC94 cases. However, the mean profiles in the lower boundary layer display larger variations between runs. This is partly caused by preventing the surface fluxes to adjust to the near-surface stability. We thus conclude that the surface boundary condition and/or the interaction of the near-surface temperature and wind-speed gradients with the surface can play a key role for the grid sensitivity at coarser grid spacings (i.e., larger than $3.125 \mathrm{~m}$ ). Note that this interaction usually strongly depends on the SGS models, as their effect is particularly strong near the surface due to the significantly smaller size of the dominant eddies close to the ground.

Nevertheless, we again see a decrease in the boundary-layer height for a finer grid spacing of $2 \mathrm{~m}$, which is clearly in line with all other experiments presented in this study. The surface conditions and the feedback with the resolved flow thus is not able to explain the grid sensitivity issue at small grid spacings.

\subsubsection{Mixing with the Free Atmosphere}

It is noteworthy that Fig. 4 indicates that for the very fine grid spacings $(2 \mathrm{~m}$ and $1 \mathrm{~m})$ the transition between the boundary layer and the free atmosphere aloft at heights of $\approx 150 \mathrm{~m}$ is rather abrupt, while for coarser grid spacings the transition is quite continuous. The sharp discontinuity might indicate that the boundary layer is decoupled from the free atmosphere so that turbulent exchange between the two ceases.

In order to asses whether changes in the mixing with the free atmosphere aloft might be responsible for the differences in the boundary-layer height and thus for the grid sensitivity, we performed a quadrant analysis (described, e.g., by Sullivan et al. 1998) of the turbulent fluxes of heat and momentum for case ILES_BC94_FIX. The results are shown for a selection of grid spacings in Fig. 8. For $\overline{w^{\prime} \theta^{\prime}}$, we see that the flux components (i.e., the fluxes within individual quadrants) decrease (in the absolute sense) linearly with height until reaching the entrainment zone. Moreover, the two negative and positive flux components are almost 

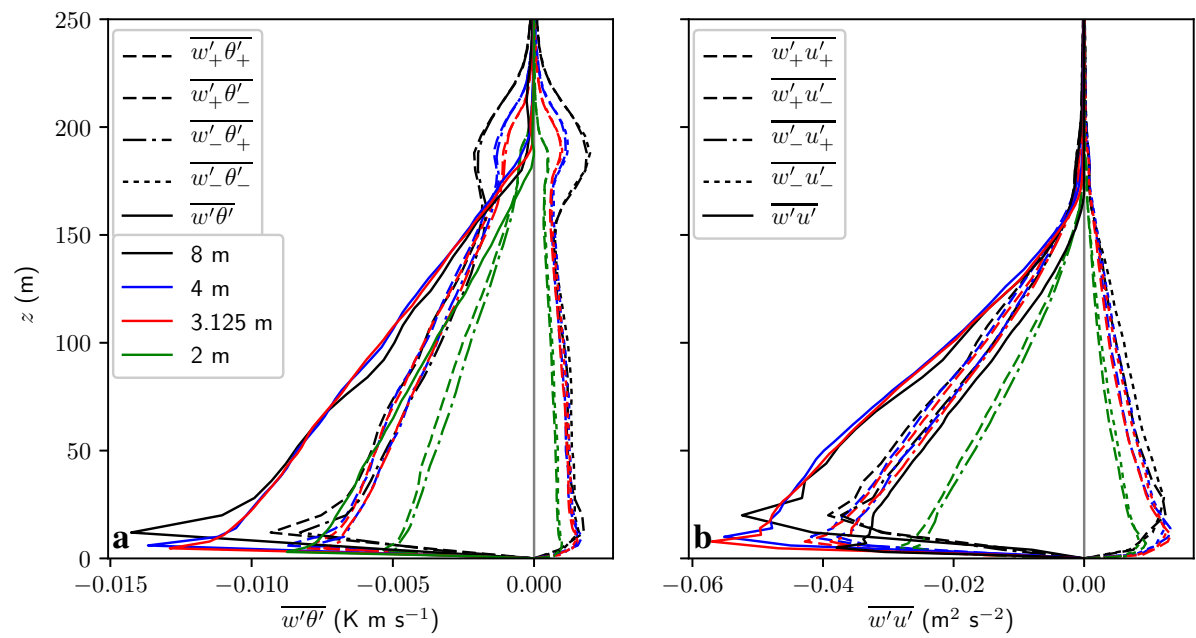

Fig. 8 Quadrant analysis for case ILES_BC94_FIX for (a) $\overline{w^{\prime} \theta^{\prime}}$ and (b) $\overline{w^{\prime} u^{\prime}}$. Note that a different color scheme is used here for improved readability

the same in the entrainment zone, suggesting that turbulent mixing can be considered to be homogeneous. Looking at the differences between grid spacings, we note similar flux components and total fluxes (the sum of the flux components) for grid spacings down to $3.125 \mathrm{~m}$, but significantly smaller (again in the absolute sense) values for the $2 \mathrm{~m}$ run. This can easily be traced back to the smaller TKE discussed above. Interestingly, we do see an entrainment peak in all components, while the total flux here is almost zero. This means that while there are exchanges of air between the boundary layer and the free atmosphere aloft, it does not lead to a net vertical transport of heat. From the available three-dimensional data we could confirm that this is related to the appearance of gravity waves at the top of the boundary layer (not shown), which explains why the quadrant analysis shows large values in individual quadrants, whereas no net transport of heat exists. This behaviour is different from the classic convective boundary layer, where entrainment continuously leads to heating of the boundary layer and cooling of the free atmosphere. As a direct consequence, LES of convective boundary layers usually only achieve a quasi-steady state. Our results here suggest that the simulated SBL under such conditions is no longer influenced by entrainment of heat from above.

Focusing on the grid sensitivity, we do see a sudden decrease of the entrainment peak for the run with 2-m grid spacing, suggesting that mixing may eventually cease for very fine grid spacings. The quadrant analysis clearly shows that the turbulent transport of heat is dominated by the surface condition as the net transport at the top of the boundary layer is negligible. This seems to imply that the entrainment of warm air is not responsible for the grid-sensitivity issue. This is also confirmed by the quadrant analysis of $\overline{w^{\prime} u^{\prime}}$ (see Fig. 8b). Generally, the findings for the heat flux can be directly applied to the momentum fluxes. The most striking difference is that we do not observe an entrainment peak in the momentum fluxes. Transport of momentum from the free atmosphere into the boundary layer is thus negligible in steady-state conditions. The flux $\overline{w^{\prime} v^{\prime}}$ is not shown as it resembles the features seen in $\overline{w^{\prime} u^{\prime}}$ and does not give additional information. 

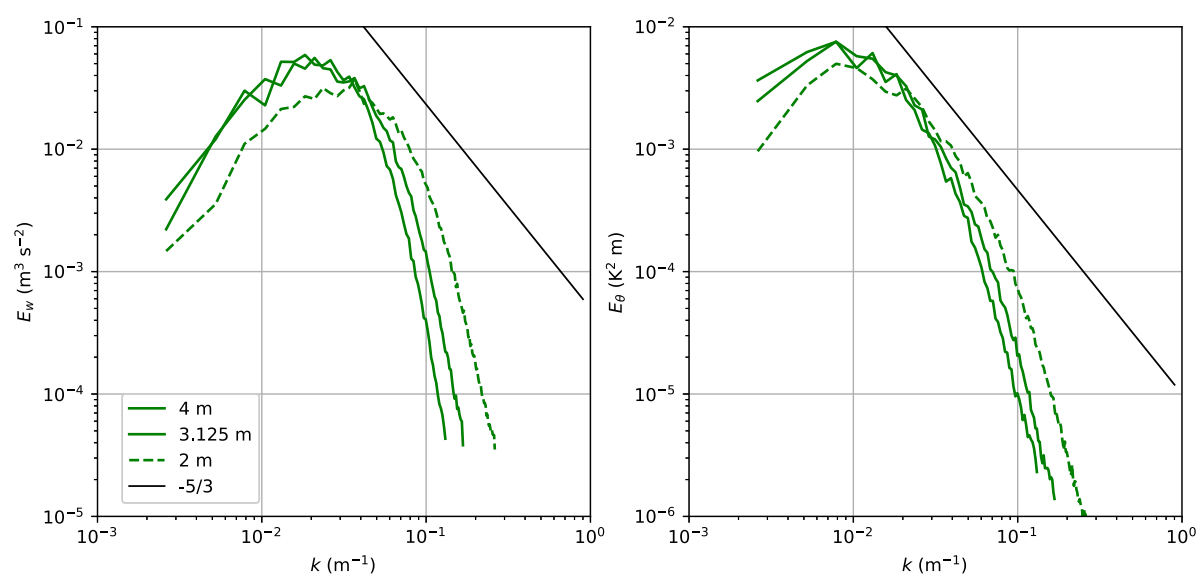

Fig. 9 Two-dimensional turbulence spectral energy density $(E)$ against wavenumber $(k)$ at a height of $50 \mathrm{~m}$ for case ILES_BC94_FIX for (a) $w$, and (b) $\theta$, calculated each $10 \mathrm{~min}$ and subsequently averaged over the last $6 \mathrm{~h}$ of simulation time

\subsubsection{Spectral Analysis}

In previous sections, we found decreased TKE for very fine grid spacings even though we would expect higher TKE for decreased grid spacings because more fine-scale turbulence is resolved. In order to better understand this issue, we calculated the turbulence spectra. Figure 9 shows the spectral energy density $(E)$ for the vertical velocity component $(w)$ and $\theta$ calculated for the grid spacings of 2-4 m for the ILES_BC94_FIX case at a height of $50 \mathrm{~m}$, which is in the middle of the boundary layer where turbulence is expected to be fully resolved. We see an increase in the spectral energy for both $w$ (and hence TKE) and $\theta$ at high wavenumbers when decreasing the grid spacings, as expected. What is surprising is the decrease in the spectral energy at the larger scales with wavenumbers in the range of $2 \times 10^{-3}-3 \times 10^{-2} \mathrm{~m}^{-1}$ when going from $\Delta=3.125 \mathrm{~m}$ to $\Delta=2 \mathrm{~m}$. We found this behaviour throughout the boundary layer (not shown), implying that the grid-sensitivity issue for the very fine spacings (i.e., going from $\Delta=3.125 \mathrm{~m}$ to $\Delta=2 \mathrm{~m}$ ) is due to changes in the large-scale turbulent motion. This seems to be in contrast to the statements of Sullivan et al. (2016), who concluded from their LESs that the sensitivity would manifest in the small scales. However, their conclusion was based on an additional run with a larger horizontal model domain which did not alter their results. Their interpretation of "small scales" thus includes the full range of the turbulence spectrum except the scales that are of size of the model domain. Our results are thus not necessarily in contradiction with their conclusions.

While the spectral analysis is only shown for the ILES_BC94_FIX cases, all simulated cases indicate that the TKE decreases when the grid spacing changes from $3.125 \mathrm{~m}$ to $2 \mathrm{~m}$ (and $1 \mathrm{~m}$ ). It is not intuitive as to why the energy content of large-scale turbulent motion decreases with decreasing grid spacings. At the moment we do not have any conclusive explanation for this behaviour. Further investigations on this phenomenon are needed, which might shed important insights into the grid-sensitivity issue. 


\section{Summary and Outlook}

One key issue for LES of the SBL that has not been solved is the grid sensitivity, i.e., the change of model results when changing the grid spacings. In this paper, we performed a comprehensive set of LES based on the GABLS1 case in order to test several processes and numerical features that have the potential for being responsible for the grid sensitivity: deficiencies in the SGS model, inaccuracies in the use of MOST for the surface boundary conditions, feedback between the surface boundary condition and the resolved near-surface temperature and wind gradients, disadvantageous properties of the numerical schemes, and mixing of heat and momentum with the free atmosphere.

Specifically, we used the original GABLS1 set-up in a standard LES configuration with the default Deardorff SGS model and performed experiments with grid spacings varying between $12.5 \mathrm{~m}$ and $1 \mathrm{~m}$. Additionally, we performed experiments where we used a modified SGS model as proposed by Dai et al. (2021). Both cases were then repeated with a nondissipative advection scheme to avoid numerical dissipation. Moreover, we ran the GABLS1 case in the ILES mode, i.e., without a SGS model, so that dissipation was provided entirely by the numerical schemes. Finally, we performed a set of sensitivity experiments based on the ILES case, where we replaced the advection scheme for temperature to improve the representation of temperature gradients near the surface and the top of the boundary layer, and additionally fixed the surface heat and momentum fluxes to avoid feedback between the surface boundary condition and the near-surface flow.

We found that the simulation period of GABLS1 in previous studies $(9 \mathrm{~h})$ is inadequate for two reasons. First, the simulated SBL is not in steady-state conditions with inertial oscillations developing after about $12.5 \mathrm{~h}$. We found that steady-state conditions are not reached before one full cycle of the inertial oscillation (i.e., not before 24-25 h). Afterwards the wind-speed profiles only change above the boundary layer (i.e., in the region of the inertial oscillation). Due to the continuous cooling of the surface, temperature profiles only reach quasi-steady state conditions. Second, depending on the model set-up the onset time of turbulence can vary on the order of several hours, which renders a comparison at $9 \mathrm{~h}$ of simulation time improper. We thus propose to use a much longer simulation period ( $36 \mathrm{~h}$ is used in our study) covering at least two to three full periods of the inertial oscillation. This also guarantees that the different turbulence initiation times have negligible effects on the thermodynamic quantities during the final analysis period. As an alternative, it might be beneficial to use an advanced method to initialize the model either by disturbing the flow field several times until turbulence sets in or via a more realistic turbulent flow field.

One of our main findings was that the grid sensitivity can be separated into two different regimes. The first regime includes grid spacings of roughly $\geq 3 \mathrm{~m}$. Here, the LES data suggest a monotonous change of the mean profiles (easily seen in the boundary-layer height). This is reproduced by all cases, even though some set-ups display faster convergence, which might thus be relevant for LES studies where only coarse grid spacings can be afforded. Note that the different model set-ups here give different converged mean profiles and fluxes in terms of the boundary-layer height and TKE. What is interesting is that we found a second regime $(\Delta<3 \mathrm{~m})$, which is characterized by a decrease in TKE and lower boundary-layer heights with further decreasing grid spacings. This finding was in line with previous LES studies (e.g., Huang and Bou-Zeid 2013; Sullivan et al. 2016; Van der Linden et al. 2019), which all displayed shallower boundary layers for increased resolution (though often considered to be negligible in these previous studies). We examined this regime for grid spacings down to $1 \mathrm{~m}$ but could not achieve grid convergence. Unfortunately, we were unable to perform 
simulations at even finer grid spacings, which is partly due to the long simulation time of $36 \mathrm{~h}$ that we considered. However, Sullivan et al. (2016) showed that the grid-sensitivity issue persists down to a model resolution of $0.39 \mathrm{~m}$, which does not promote optimism that convergence could be easily reached. Note that the regime separation around grid spacings of $3 \mathrm{~m}$ is probably only valid for the GABLS1 set-up. It would be interesting to test this finding for other scenarios with different static and dynamic stability. In that scope it would make sense to make the results dimensionless in search of a more universal parameter (e.g., Ozmidov length, outer length scale) indicating at what resolution the regime change (and possibly grid convergence) is reached.

Another key result is that the proposed SGS model by Dai et al. (2021), though reducing the grid sensitivity for coarser grid spacings and thus being a significant improvement, does not fully resolve the grid-sensitivity issue. Also, numerical dissipation of the advection scheme seems to be an important compounding factor. Interestingly, all cases tend to predict very similar boundary-layer heights at a grid spacing of $1 \mathrm{~m}$, including the ILES case, where the SGS model is switched off. This is nicely summarized in Fig. 10. It is striking that for almost all cases, we observe a maximum in the boundary-layer height around grid spacings of 3$4 \mathrm{~m}$, which is in agreement with the discussion above (except D80-R_PW70 cases which display monotonously decreasing boundary-layer heights with decreasing grid spacings). For coarser grid spacings, the cases display heterogeneous behaviours, supporting the finding that some configurations converge faster towards the maximum value than others. In particular, it is noteworthy that the D80-R scheme displays a much weaker grid sensitivity compared to all other cases. For the second regime $(\Delta \leq 3.125 \mathrm{~m})$, all cases reveal a decrease in the boundary-layer height. With a grid spacing of $1 \mathrm{~m}$, all runs predict almost the same boundarylayer height.

In order to avoid compounding effects of numerical dissipation and dissipation provided by the SGS model, and to investigate which other processes might be responsible for the grid sensitivity, we analyzed three cases: case ILES using the WS02 advection scheme (high numerical dissipation, low dispersion error); case ILES_BC94, using the boundary condition scheme for temperature instead of the WS02 scheme, which can better represent vertical temperature gradients but comes with a several computational penalty; and case ILES_BC94_FIC, which is based on ILES_BC94 but with fixed surface fluxes. We demonstrate that all processes have a significant impact on the profiles of mean quantities and turbulent fluxes. The changes we implemented, however, cannot fully resolve the gridsensitivity issue. In the future it might be possible to gain more insights into the issue by using grid-refinement techniques, such as adaptive mesh refinement or the self-nesting capability of PALM (e.g., van Hooft et al. 2018a, b; Hellsten et al. 2021).

In summary, this work illustrates that the cause of the grid-sensitivity issue for LES of the SBL remains unknown, especially for fine grid spacings. One possible avenue for further analysis is to separate cause and effect. The most striking feature was shown to be the lower boundary-layer heights and the decreases in TKE for smaller grid spacings (e.g., from $3.125 \mathrm{~m}$ to $1 \mathrm{~m}$ ). The two are clearly related as less TKE indicates less mixing and thus less capability of the SBL to penetrate into the free atmosphere before reaching a steady state. While we did not show this explicitly, we find that the TKE is reduced throughout the boundary layer. Using turbulence spectra, we show that energy is removed from the largest scales in the production range, while there is energy gain in small scales with higher resolutions. The reason for this decrease in TKE remains unknown. Future investigations on the TKE budgets, similar to the study by Heinze et al. (2015) under convective conditions, might elucidate why the energy content of large-scale turbulence is altered. 


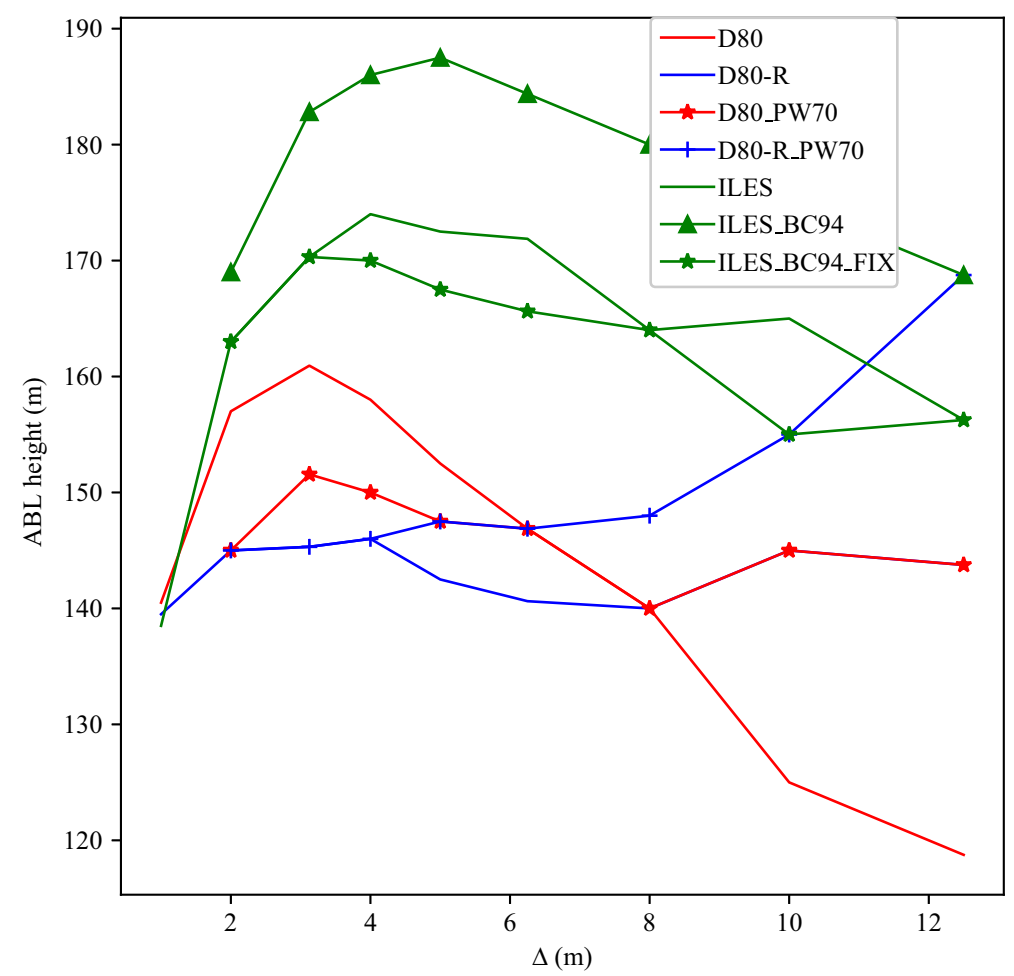

Fig. 10 Boundary-layer height against grid spacing for all simulated cases. The boundary-layer height was calculated based on the height of the maximum wind speed. Note that data for 1-m grid spacing were only available for the baseline runs D80, D80-R, and ILES

Last but not least, there is no agreed quantitative measure of grid convergence. Hence, claims of grid convergence by many studies are somewhat arbitrary. To develop quantitative measures of grid convergence we need the 'truth'. Direct numerical simulations of the SBL, although with low Reynolds number, are potentially very useful to serve as the benchmark especially when properly non-dimensionalized (e.g., Nieuwstadt 2005; van de Wiel et al. 2008; Ansorge and Mellado 2014). While this is not specifically addressed here, it is important to stress again that the grid-sensitivity issue seems to persist (visually) in many studies that report grid convergence, and benchmark cases are strongly needed to evaluate grid convergence.

Acknowledgements We would like to thank the two anonymous reviewers for many helpful suggestions to improve the paper. Furthermore, we are grateful to Yi Dai, Sukanta Basu, and Stephan de Roode (all at Delft University of Technology, the Netherlands) and particularly Siegfried Raasch (Leibniz University Hannover) for discussions on the topic. DL acknowledges support from the U.S. National Science Foundation under Grant AGS-1853354 and the Alexander von Humboldt Foundation which supported his visit to Leibniz University Hannover. Simulations were carried out on the computer clusters of the North-German Supercomputing Alliance (HLRN).

Funding Open access funding provided by University of Bergen (incl Haukeland University Hospital).

Open Access This article is licensed under a Creative Commons Attribution 4.0 International License, which permits use, sharing, adaptation, distribution and reproduction in any medium or format, as long as you give 
appropriate credit to the original author(s) and the source, provide a link to the Creative Commons licence, and indicate if changes were made. The images or other third party material in this article are included in the article's Creative Commons licence, unless indicated otherwise in a credit line to the material. If material is not included in the article's Creative Commons licence and your intended use is not permitted by statutory regulation or exceeds the permitted use, you will need to obtain permission directly from the copyright holder. To view a copy of this licence, visit http://creativecommons.org/licenses/by/4.0/.

\section{Appendix: Effect of Model Numerics on Turbulence Spectra}

The effect of the different numerical schemes on turbulence spectra are illustrated in Fig. 11. Case D80 displays a severe drop of spectral energy density at high wavenumbers that correspond to wavelengths of $\leq 6 \Delta$, which is caused by high numerical dissipation of the WS02 scheme. In case D80_PW70, where the non-dissipative PW70 scheme is employed, the dropoff happens at higher wavenumbers and is solely caused by the SGS model. As a result, more energy is contained in the smallest scales in case D80_PW70 compared to case D80, while energy in the production and inertial subranges is higher for case D80.

In case ILES, the drop of energy amount at the highest wavenumbers is evidently weaker than in case D80 as a consequence of not having an SGS model dissipating energy. Due to the numerical dissipation provided by the WS02 scheme, however, there is still sufficient dissipation. While we observe comparable spectral energy density between case ILES and case D80 in the production and inertial subranges for the vertical velocity, we note higher spectral energy density in the inertial subrange for temperature. Finally, for case ILES_BC94 we observe almost no change in the vertical velocity spectrum but changes in the temperature spectrum when compared against case ILES. This is because the BC94 scheme is only applied to scalars like temperature. Specifically, case ILES_BC94 displays lower spectral energy density in the inertial subrange and the dissipation range of the temperature spectrum, which suggests that the $\mathrm{BC}$ scheme has even more numerical dissipation than the WSO2 scheme.
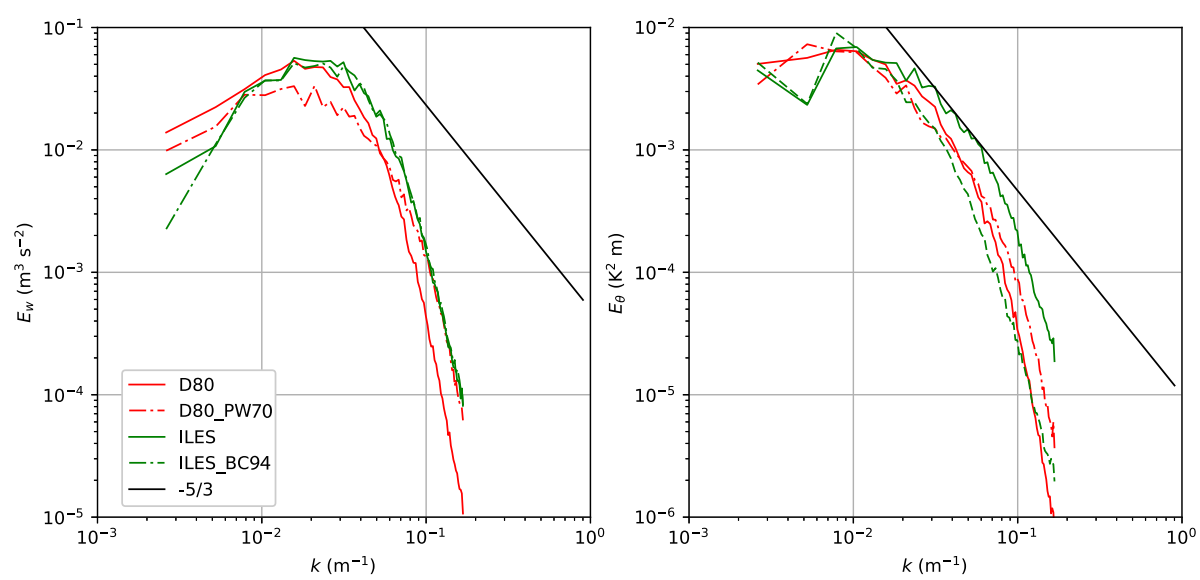

Fig. 11 As Fig. 9 but for cases D80, D80_PW70, ILES, and ILES_BC94 with a grid spacing of 3.125m 


\section{References}

Adams NA, Hickel S (2009) Implicit large-eddy simulation: theory and application. In: Eckhardt B (ed) Advances in turbulence XII. Springer Berlin Heidelberg, Berlin, pp 743-750

Ansorge C, Mellado JP (2014) Global intermittency and collapsing turbulence in the stratified planetary boundary layer. Boundary-Layer Meteorol 153:89-116. https://doi.org/10.1007/s10546-014-9941-3

Basu S, Porte-Agel F (2006) Large-eddy simulation of stably stratified atmospheric boundary layer turbulence: a scale-dependent dynamic modeling approach. J Atmos Sci 63:2074-2091

Beare RJ, Cortes MAJ, Cuxart J, Esau I, Golaz C, Holtslag AAM, Khairoutdinov M, Kosovic B, Lewellen D, Lund T, Lundquist J, Mccabe A, Macvean MK, Moene A, Noh Y, Poulos G, Raasch S, Sullivan P (2006) An intercomparison of large-eddy simulations of the stable boundary layer. Boundary-Layer Meteorol 118:247-272

Chlond A (1994) Locally modified version of Bott's advection scheme. Mon Weather Rev 122:111-125

Dai Y, Basu S, Maronga B, de Roode SR (2021) Addressing the grid-size sensitivity issue in large-eddy simulations of stable boundary layers. Boundary-Layer Meteorol 178:63-89. https://doi.org/10.1007/ s10546-020-00558-1

Deardorff JW (1980) Stratocumulus-capped mixed layers derived from a three-dimensional model. BoundaryLayer Meteorol 18:495-527

Derbyshire SH (1990) Nieuwstadt's stable boundary layer revisited. Q J R Meteorol Soc 116:127-158

Donda JMM, van Hooijdonk IGS, Moene AF, Jonker HJJ, van Heijst GJF, Clercx HJH, van de Wiel BJH (2015) Collapse of turbulence in stably stratified channel flow: a transient phenomenon. Q J R Meteorol Soc 141:2137-2147. https://doi.org/10.1002/qj.2511

Fernando HJS, Weil JC (2010) Whither the stable boundary layer? A shift in the research agenda. Bull Am Meteor Soc 91:1475-1484

Heinze R, Mironov D, Raasch S (2015) Second-moment budgets in cloud-topped boundary layers: a large-eddy simulation study. J Adv Model Earth Syst. https://doi.org/10.1002/2014MS000376

Hellsten A, Ketelsen K, Sühring M, Auvinen M, Maronga B, Knigge C, Barmpas F, Tsegas G, Moussiopoulos $\mathrm{N}$, Raasch S (2021) A nested multi-scale system implemented in the large-eddy simulation model PALM model system 6.0. Geosci Model Dev Discuss. https://doi.org/10.1002/qj.2511

Hoffmann F (2017) On the limits of Köhler activation theory: how do collision and coalescence affect the activation of aerosols? Atmos Chem Phys. https://doi.org/10.5194/acp-17-8343-2017

Huang J, Bou-Zeid E (2013) Turbulence and vertical fluxes in the stable atmospheric boundary layer. Part i: A large-eddy simulation study. J Atmos Sci 70:1513-1527. https://doi.org/10.1175/JAS-D-12-0167.1

Knigge C, Auerswald T, Raasch S, Bange J (2015) Comparison of two different methods that provide highly resolved atmospheric turbulence data for simulations of stall effects. Comp Fluids 108:57-66. https:// doi.org/10.1016/j.compfluid.2014.11.005

Lu H, Porte-Agel F (2013) A modulated gradient model for scalar transport in large-eddy simulation of the atmospheric boundary layer. Phys Fluids 25(015):110. https://doi.org/10.1063/1.4774342

Maronga B, Bosveld FC (2017) Key parameters for the life cycle of nocturnal radiation fog: a comprehensive large-eddy simulation study. Q J R Meteorol Soc 143:2463-2480. https://doi.org/10.1002/qj.3100

Maronga B, Gryschka M, Heinze R, Hoffmann F, Kanani-Sühring F, Keck M, Ketelsen K, Letzel MO, Sühring M, Raasch S (2015) The parallelized large-eddy simulation model (PALM) version 4.0 for atmospheric and oceanic flows: Model formulation, recent developments, and future perspectives. Geosci Model Dev 8:2515-2551. https://doi.org/10.5194/gmd-8-2515-2015

Maronga B, Banzhaf S, Burmeister C, Esch T, Forkel R, Fröhlich D, Fuka V, Gehrke K, Geletic J, Giersch S, Gronemeier T, Groß G, Heldens W, Hellsten A, Hoffmann F, Inagaki A, Kadasch E, Kanani-Sühring F, Ketelsen K, Khan BA, Knigge C, Knoop H, Krc P, Kurppa M, Maamari H, Matzarakis A, Mauder M, Pallasch M, Pavlik D, Pfafferott J, Resler J, Rissmann S, Russo E, Salim M, Schrempf M, Schwenkel J, Seckmeyer G, Schubert S, Sühring M, von Tils R, Vollmer L, Ward S, Witha B, Wurps H, Zeidler J, Raasch S (2020a) Overview of the PALM model system 6.0. Geosci Model Dev 13:1335-1372. https:// doi.org/10.5194/gmd-2019-103

Maronga B, Knigge C, Raasch S (2020b) An improved surface boundary condition for large eddy simulations based on Monin-Obukhov similarity theory: Evaluation and consequences for grid convergence in neutral and stable conditions. Boundary-Layer Meteorol 174:297-325. https://doi.org/10.1007/s10546019-00485-w

Momen M, Bou-Zeid E (2017) Mean and turbulence dynamics in unsteady Ekman boundary layers. J Fluid Mech. https://doi.org/10.1017/jfm.2017.76

Monin AS, Obukhov AM (1954) Basic laws of turbulent mixing in the atmosphere near the ground. Tr Akad Nauk SSSR Geofiz Inst 24:163-187 
Nieuwstadt FTM (2005) Direct numerical simulation of stable channel flow at large stability. Boundary-Layer Meteorol 116:277-299

Piacsek SA, Williams GP (1970) Conversion properties of convection difference schemes. J Comput Phys 198:580-616

Pope S (2000) Turbulent flows. Cambridge University Press, Cambridge

Raasch S, Franke T (2011) Structure and formation of dust-devil-like vortices in the atmospheric boundary layer-a high resolution numerical study. J Geophys Res 116

Smolarkiewicz P, Margolin L, Wyszogrodzki A (2007) Implicit large-eddy simulation in meteorology: from boundary layers to climate. J Fluids Eng 129:1533. https://doi.org/10.1115/1.2801678

Stoll R, Porte-Agel F (2008) Large-eddy simulation of the stable atmospheric boundary layer using dynamic models with different averaging schemes. Boundary-Layer Meteorol 126:1-28

Sullivan PP, Moeng CH, Stevens B, Lenschow DH, Mayor SD (1998) Structure of the entrainment zone capping the convective atmospheric boundary layer. J Atmos Sci 55:3042-3064

Sullivan PP, Weil JC, Patton EG, Jonker HJJ, Mironov DV (2016) Turbulent winds and temperature fronts in large-eddy simulations of the stable atmospheric boundary layer. J Atmos Sci 73:1815-1840. https://doi. org/10.1175/JAS-D-15-0339.1

van de Wiel BJH, Moene AF, De Ronde WH, Jonker HJJ (2008) Local similarity in the stable boundary layer and mixing-length approaches: consistency of concepts. Boundary-Layer Meteorol 128:103-116. https://doi.org/10.1007/s10546-008-9277-y

Van der Linden SJA, Edwards JM, van Heerwaarden CC, Vignon E, Genthon C, Petenko I, Baas P, Jonker HJJ, van de Wiel CJH (2019) Large-eddy simulations of the steady wintertime Antarctic boundary layer. Boundary-Layer Meteorol 173:165-192. https://doi.org/10.1007/s10546-019-00461-4

van Hooft JA, Popinet S, van de Wiel BJH (2018a) Adaptive cartesian meshes for atmospheric single-column models: a study using basilisk 18-02-16. Geosci Model Dev 11:4727-4738. https://doi.org/10.5194/ gmd-11-4727-2018

van Hooft JA, Popinet S, van Heerwaarden CC, van der Linden SJA, de Roode SR, van de Wiel BJH (2018b) Towards adaptive grids for atmospheric boundary-layer simulations. Boundary-Layer Meteorol 167:421443. https://doi.org/10.1007/s10546-018-0335-9

van Stratrum BJH, Stevens B (2015) The influence of misrepresenting the nocturnal boundary layer on idealized daytime convection in large-eddy simulation. J Adv Model Earth Syst 7:423-436

Wicker LJ, Skamarock WC (2002) Time-splitting methods for elastic models using forward time schemes. Mon Weather Rev 130:2088-2097

Williamson JH (1980) Low-storage Runge-Kutta schemes. J Comput Phys 35:48-56

Publisher's Note Springer Nature remains neutral with regard to jurisdictional claims in published maps and institutional affiliations. 